\title{
Cross-Cultural Adaptation of the Physician Orders for Life-Sustaining Treatment Form to Brazil
}

\author{
Vania F.S. Mayoral, MD, MPH, Fernanda B. Fukushima, MD, PhD, Aniela M. Rodrigues, MD, ${ }^{1}$ \\ Raissa P. Carvalho, MD, Larissa P. Carvalho, MD, Leandro A.F.V. Pinheiro, BF, \\ Bertha F. Polegato, MD, PhD, ${ }^{1}$ Marcos F. Minicucci, MD, PhD, ${ }^{1}$ Rick Bassett, RN, MSN, \\ Alvin H. Moss, MD, Karl E. Steinberg, MD, CMD, and Edison I.O. Vidal, MD, MPH, $\mathrm{PhD}^{1}$
}

\begin{abstract}
Background: The Physician Orders for Life-Sustaining Treatment (POLST) paradigm is considered one of the most important strategies to respect patients' values at the end of life in the United States. The cross-cultural adaptation of POLST entailed several methodological considerations, which may be informative for international researchers who may also consider bringing POLST to their countries as a means to promote care at the end of life that is consistent with patients' preferences.

Objective: To report the methods and outcome of the cross-cultural adaptation of the POLST form to Brazil. Design: Cross-cultural adaptation study.

Setting/Subjects: Twenty physicians and 10 patients at a university hospital participated in the pilot tests.

Results: The cross-cultural adaptation process included choosing which existing POLST form(s) to use as a source, deciding the intended reading level, which healthcare professionals should be allowed to sign the form, and consultation with attorneys, bioethicists, and members of the National POLST Paradigm Task Force. Pilot tests occurred in two stages using different approaches. First, 20 physicians were trained about POLST and asked for any unclear aspects related to the form. Second, trained investigators completed POLST forms after engaging in advance care planning conversations with 10 hospitalized patients or patients' surrogates.

Conclusions: This report provides a basis for future cross-cultural adaptations of POLST to other countries. The authors hope such new adaptations will broaden the possibilities of research using POLST and also may promote wider provision of care at the end of life that is consistent with patients' preferences.
\end{abstract}

Keywords: advance care planning; advance directives; Brazil; cross-cultural adaptation studies; palliative care; POLST

\section{Introduction}

$\mathbf{T}$ He Physician Orders for Life-Sustaining Treatment (POLST) paradigm (www.polst.org) represents a coordinated system to elicit patients' end-of-life preferences of care and to translate those values into a set of medical orders concerning desired goal-directed treatments. ${ }^{1,2}$ A central component of that paradigm is the POLST form, which communicates the treatment preferences of patients as a standardized set of medical orders. Over the last decades
POLST has become one of the most important strategies to honor patients' preferences of care at the end of life in the United States. $^{3,4}$

In Brazil it is still uncommon for most healthcare professionals to engage in advance care planning conversations with patients who suffer from serious chronic illnesses or frailty. In an unpublished study by researchers from the Barretos Cancer Hospital (state of São Paulo), of the medical records of 1284 oncologic patients, chart review found rare documentation of advance care planning discussions (personal communication

\footnotetext{
${ }^{1}$ Internal Medicine Department, ${ }^{2}$ Anesthesiology Department, Botucatu Medical School, Sao Paolo State University (UNESP), Botucatu, Sao Paolo, Brazil.

${ }^{3}$ Campinas Federation of Social Organizations-FEAC, Campinas, Brazil.

${ }_{5}^{4}$ Center for Nursing Excellence, St. Luke's Health System, Boise, Idaho.

${ }^{5}$ Center for Health Ethics and Law, West Virginia University, Morgantown, West Virginia.

${ }^{6}$ Institute for Palliative Care, California State University, Oceanside, California.

Accepted January 15, 2018.
} 
from Prof. Bianca S. R. Paiva). Furthermore, in another Brazilian study $74 \%$ of a sample of healthcare professionals from four hospitals in the city of São Paulo were not familiar with the concept of advance directives, a frequent outcome of advance care planning. ${ }^{5}$ The lack of advance care planning characterizes a severe shortcoming of the current Brazilian healthcare system, which is likely to be present in many other countries. ${ }^{6-8}$ We realized that promoting the implementation of POLST in Brazil could represent an important step to change that picture for the better. Therefore, we proposed the cross-cultural adaptation of the POLST form to Brazil as a first movement in that direction.

Because the cross-cultural adaptation of the POLST form entailed several methodological considerations when compared with that of other instruments, we present the current report aiming to inform international researchers and clinicians, who may also consider bringing POLST to their countries as a means to promote the provision of care at the end of life that is consistent with patients' values.

\section{Methods}

The methodology was based upon the recommendations of the International Society of Pharmacoeconomics and Outcomes Research. ${ }^{9}$ We describe below the steps involved in the study.

(1) Preparation. During this stage we obtained authorization from the National POLST Paradigm Task Force. ${ }^{10}$ Because there are many different versions of POLST forms in use throughout the United States, this stage also involved the decision concerning which form(s) to use as the source for adaptation.

(2) Forward translation. Three independent nativespeaking Brazilians fluent in English performed three translations of the reference form into Brazilian Portuguese. Two of those translators were physicians, whereas the third translator was a lay Brazilian English teacher.

(3) Reconciliation. A committee assessed the forward translations and arrived at a consensual single version of the form in Brazilian Portuguese. Three geriatricians, two palliative care physicians, two internists, three medical students, and a layperson representing patients/families composed that committee.

(4) Back translation. Two native-speaking Americans with fluency in Brazilian Portuguese performed two independent back translations into American English. Both translators were English teachers living in Brazil.

(5) Back-translation review. Three researchers and a member of the National POLST Paradigm Task Force examined the back translations and revised the version derived from the reconciliation meeting.

(6) Cognitive debriefing/Pilot testing. We merged the pilot test phase with its review in an iterative process, so that we revised the form according to the comments of the research subjects of each round of interviews before proceeding to the next.

This phase was approached in two sequential stages. In the first stage we pilot tested the Brazilian form by means of individual training sessions to groups of 10 physicians regarding the content of the POLST form and how to engage with patients in a conversation that enables the completion of the form. In the second stage, four interviewers who had undergone a similar training performed individual interviews with a group of 10 different patients or patients' surrogates at the public university hospital where the research was based.

Physicians selected for the first stage of the pilot test were eligible if they provided care to patients with serious illness in their daily practice and had not been involved with previous phases of this study (e.g., as translators or members of the reconciliation committee). The selection criteria for patients in the second stage of the pilot test were: (1) age of 21 years or older, (2) hospitalization at the study hospital, (3) a "No, I would not be surprised" response from one of their physicians to the question "Would you be surprised if the patient died within one year?," and (4) decision-making capacity during the interview according to established standards. ${ }^{11}$ If a patient fulfilled the first three criteria but did not have decision-making capacity, a surrogate was invited to participate in the study.

For the first stage, the physicians who were interviewed were specifically asked regarding items that were not culturally sound or unclear and their doubts concerning the advance care planning conversation. Because the POLST form is a medical order form that is completed by a healthcare professional (i.e., not by the patient), during the second stage of the pilot test the interviewers were asked about any unclear aspect of the form or any difficulties arising during the conversation with patients or surrogates. Interviewers were instructed to confirm that patients had correct understanding of the process several times during the interview. The researchers captured all comments from participants in the cognitive debriefing/pilot study by handwritten annotations over the POLST forms under adaptation and in complementary sheets as needed.

(7) Presentation of the form to members of the National POLST Paradigm Task Force, an attorney, and two bioethicists. The study coordinator had an in-person meeting with a member of the National POLST Paradigm Task Force to discuss the status of the cross-cultural adaptation process. Afterward, the researchers presented the form under adaptation to an attorney specialized in advance directives and to two bioethicists in Brazil.

(8) Presentation of the form to the POLST Developing State Assistance Committee. The researchers produced another back translation of the form and presented it to the POLST Developing State Assistance Committee by means of a web conference.

(9) Proofreading and final report. Researchers checked the form for minor print errors and produced a detailed report in Brazilian Portuguese concerning each adaptation decision that was made throughout the study (available upon request).

We did not examine statistical aspects such as Cronbach's $\alpha$ or factor analysis because, given the nature of the POLST form, they would be inappropriate. ${ }^{12}$

We obtained approval from the local ethics committee. All study subjects provided written informed consent for participation. 


\section{Results}

Considering the length of the POLST form (over 1000 words) and space restrictions of this article, it is not possible to present in detail every translation and cultural adaptation choice that was made. Instead, this article provides readers with the most important decisions that were made to produce a culturally appropriate POLST form that still complied with the standards established by the National POLST Paradigm Task Force.

Following the advice of a member of the National POLST Paradigm Task Force and the scrutiny of several POLST forms, we chose to use the 2014 version of the POLST form from the state of Oregon as our primary source for the forward translation phase. That advice was based on the fact that there are different POLST forms in use in different states in the United States and that those forms change over time according to feedback received from their users. The researchers chose the Oregon POLST form as the primary reference because that state had just released its updated form and because Oregon was the state with the oldest POLST program in the United States. However, because a significant portion of the instructions on the back of the Oregon form were related to the POLST registry specific to that state, the 2014 version of the California POLST form was used as a reference for that section. The links to the source versions of the Oregon and California POLST forms, the final version of the form adapted to Brazil, and the final back translation are provided in the Supplementary Appendix (Supplementary Data are available online at www.liebertpub.com/jpm).

Reading level of the form was the next important decision that we faced. This was an important consideration because in Brazil there is still a large proportion of the population who is illiterate or with less than four years of schooling, especially among older people. The reasoning was that, although patients are expected to receive copies of their POLST forms, the form is actually a part of their medical records and is primarily intended to communicate a set of medical orders to other healthcare professionals. Hence, the level of language that is found in medical records was chosen and healthcare professionals were instructed that it is their duty to conduct the advance care planning conversations according to the level of health literacy of the patient.

The word "order" in the title "Physician Orders for LifeSustaining Treatment" in English was adapted to "prescrição" (exact translation of "prescription"), because in Brazilian Portuguese the word "ordem" (exact translation of "order") is rarely used in the medical context and has some unwanted cultural connotations (e.g., arrogance, power over and hierarchy), which could be misinterpreted by other physicians assessing the patient. On the other hand, the word "prescrição" is commonly used and conveys the intended message that a physician assessed the patient and takes responsibility for the actions he or she has prescribed.

Another important issue that we faced concerned which healthcare professionals should be allowed to sign Brazilian POLST forms. Although in several states of the United States, physicians, nurse practitioners, and physician assistants are allowed to sign POLST forms, the reconciliation committee was unanimous that at this moment only physicians should be allowed to sign Brazilian POLST forms. This decision was based on the fact that in the current Brazilian healthcare cultural context, only physicians make "do not resuscitate orders" and that, even among palliative care teams, advance care planning conversations are considered a responsibility of physicians. Furthermore, the committee understood that allowing other healthcare professionals to sign Brazilian POLST forms would jeopardize the chances of the Brazilian Federal Medical Council endorsing POLST in Brazil in the near future.

The pilot test with the first group of 10 physicians included 1 nephrologist, 1 oncologist, 2 intensive care physicians, 2 palliative care specialists, 1 hematologist, 1 gastroenterologist, and 2 internists. Their mean time since medical school graduation was 20 years. The following issues raised by these physicians deserve notice. One physician manifested concerns that principles of POLST could be incompatible with Brazilian regulations and that it would always be safer from a legal perspective to perform the most aggressive potentially life-sustaining interventions. Two physicians were unclear on the difference between the POLST form and advance directives. Two physicians stated that the option for "limited treatment" in section B of the form should specify clearly if central intravascular lines were also included under that option because in the Brazilian medical community, central lines may be perceived by some as an excessively invasive intervention. Therefore, section B of the POLST form was revised to add central lines under "limited treatment," and a description of two regulations from the Brazilian Federal Medical Council that provide a basis for the withholding/ withdrawal of treatments at the end of life and for advance directives was added on the back of the form.

During the presentation of the form to the second group of 10 physicians, there was no further need to change it because of other cultural issues or lack of clarity, although 3 physicians still felt unsure about the legal permissibility of withholding/withdrawing life-sustaining treatment, despite having understood the text concerning those regulations. That second group included one hematologist, one gastroenterologist, one nephrologist, one palliative care clinician, one intensive care physician, and five internists. Their mean time since graduation was 15.8 years.

The second stage of the pilot test involved the interview of seven patients and three surrogates. The mean age of all 10 patients was 73.4 years, and they had an average of 4.7 years of schooling. Five patients were women. The three representatives were patients' daughters and their ages ranged from 37 to 55 years. Patients had a median Karnofsky Performance Status of $45 \%$ at the time of the interview and had an average of seven diagnoses. Their main reason for admission was decompensated heart failure for four patients, delirium and pneumonia for two patients each, and decompensated chronic obstructive pulmonary disease and bone metastases for one patient each. None of the four interviewers reported lack of clarity associated with completing the form during the interview with patients or surrogates. All patients and surrogates welcomed the advance care planning conversation and reported that they felt comfortable during the process. One patient spontaneously asked the interviewer to inform her attending physicians regarding her preferences of care.

The in-person meeting with a member of the National POLST Paradigm Task Force highlighted the importance of not mingling the concepts of POLST and advance directives. The POLST form is an advance care planning document, 
where the values of patients with serious illness are translated through shared decision making into a set of medical orders concerning cardiopulmonary resuscitation and other medical interventions. That meeting also directed us to some invaluable resources for our project, such as the 2014 POLST legislative guide. ${ }^{13}$

The consultation with an attorney specialized in advance directives called our attention to problems concerning the translation of the definition of healthcare representative from the American to the Brazilian context. After further legal advice, we understood that in Brazil there was no clear law or regulation defining "healthcare representative," so we decided to exclude any attempt to define it within the form to decrease the chances that its content could prompt legal action or delay its approval. At that attorney's suggestion, we also added a brief explanation about POLST below the form heading.

The discussion with two bioethicists did not disclose any bioethical flaws or lead to any change in the form. However, it was important because it reinforced the importance of presenting the concept of POLST to the Federal Medical Council in Brazil before attempting to start a pilot POLST program.

\section{Discussion}

The process of cross-cultural adaptation of the POLST form involved a few methodological considerations before and during its implementation because of some particularities of that form when compared with other instruments. For instance, because there is not a single POLST form, we had to choose which source form to use. Additionally, the POLST form is neither a questionnaire nor an instrument designed primarily for epidemiological research, but essentially a standardized medical order document intended for pragmatic purposes related to the care of patients. The form is not given to patients for them to fill in their preferences of care, but rather healthcare professionals complete it based on one or more advance care planning conversations with patients. Because of those features we decided to implement two different strategies for pilot testing the form, which involved both the training of physicians about POLST and patient engagement in advance care planning conversations so that researchers could check for confusing aspects and inconsistencies concerning the form. Moreover, the adaptation of the form required considerations such as its agreement with local laws and regulations and the knowledge/misconceptions of healthcare professionals as to those regulations.

The debate concerning advance directives began in the United States more than 50 years ago. ${ }^{14}$ Over the last few decades several countries have initiated discussions and enacted regulations concerning advance directives. ${ }^{15-17}$ Those regulations attempt to address the common question of how to respect the autonomy of patients in situations where they have lost their capacity to consent to or refuse medical treatments. As countries move forward with such discussions and policies, some of them may determine that written advance directives might not be sufficient to ensure that patients' values and preferences of care are respected at the end of their lives. As a matter of fact that was the conclusion of several researchers regarding the effectiveness of advance directives in the United States. ${ }^{18-22}$ POLST was created with the intent to increase the awareness of the patient's advance care planning wishes and the chances that these preferences are honored at the end of their lives. A recent systematic review concluded that, despite several gaps in the research about POLST, there is strong evidence that medical orders documented in POLST forms influence the kinds of treatments provided to patients at the close of life. ${ }^{4}$ Therefore, POLST has the potential to become an important asset for the promotion of advance care planning conversations and to facilitate the respect of patients' preferences regarding medical treatments at the end of life in a variety of countries that are already interested in those matters.

As guidance for future adaptations of POLST to other countries, we would recommend the following principles: (1) to go beyond simple translations and perform formal multistep cross-cultural adaption processes; (2) to engage in partnership with the National POLST Paradigm Task Force early on; (3) to fully understand the differences between POLST and advance directives, including the kind of regulatory support needed in each context; (4) to use language compatible with that of medical records; (5) not to treat the POLST form as if it were a questionnaire administered to patients; (6) to use multistep pilot tests involving healthcare professionals, patients, and surrogates; and (7) to consider any applicable, venue-specific regulations regarding healthcare representatives/surrogates.

There are several potential limitations of this study. Although all translators who worked in this study were native speakers of the target language and fluent in the reference language, none of them were certified professional translators. It was not possible for the bioethics specialists and the attorney that we consulted to participate in the reconciliation committee convened for the assessment of the three forward translations. It was also not practical to convene multiple meetings of that committee to adjudicate every change of the form under adaptation during the later stages of the project. We did not perform another pilot test with the final version of the form as we had done after the review of the first back translations. However, our methods and report were in harmony with accepted guidelines. ${ }^{9,23-26}$ Finally, our study was restricted to one center in Brazil. However, despite the country's large geographical dimensions, all of its regions share the same language, and cross-cultural adaptations of other instruments conducted in one region are usually considered appropriate for the whole country. ${ }^{27-29}$ The following are strengths of the study: (1) including a layperson in the reconciliation committee; (2) reviewing the feedback from the pilot tests in an iterative process; and (3) engaging in conversations with members of the National POLST Paradigm Task Force throughout the crosscultural adaptation process.

In summary, this report provides a basis for future crosscultural adaptations of the POLST paradigm to other countries. The authors hope such new adaptations will broaden the possibilities of research using POLST and also may promote wider provision of care at the end of life that is consistent with patients' preferences.

\section{Acknowledgments}

The authors wish to express their gratitude to those who helped them in several stages of this work: Amy Vandenbroucke, Alessandro Jacinto, Bill Pfunder, Erik Fromme, Judy Black, Judy Thomas, Hanna Nelson, Luciana Dadalto, 
Margie Carley, Paulo Villas Boas, Reinaldo Ayer de Oliveira, Susan Nelson, Toshio Chiba, and W. Saad Hossner. This work was supported by São Paulo Research Foundation (FAPESP) and by the Brazilian National Council for Scientific and Technological Development (CNPq). Sponsors did not play any role in the conception, design, or conduction of the study, and were neither involved in the analysis/interpretation of data nor in the preparation of the article.

\section{Authors Disclosure Statement}

No competing financial interests exist.

\section{References}

1. Sabatino CP: The evolution of health care advance planning law and policy. Milbank Q 2010;88:211-239.

2. Bomba PA, Kemp M, Black JS: POLST: An improvement over traditional advance directives. Cleve Clin J Med 2012; 79:457-464.

3. Institute of Medicine (U.S.), (ed): Dying in America: Improving Quality and Honoring Individual Preferences near the End of Life. Washington, DC: The National Academies Press, 2015.

4. Hickman SE, Keevern E, Hammes BJ: Use of the physician orders for life-sustaining treatment program in the clinical setting: A systematic review of the literature. J Am Geriatr Soc 2015;63:341-350.

5. Rossini RCCC, Oliveira VI, Fumis RRL: The living will: health care professionals are unaware of its importance. RBM Rev Bras Med 2013;70:4-8.

6. Murray S: 2015 Quality of Death Report. www.lien foundation.org/sites/default/files/2015\%20Quality\%20of\% 20Death\%20Report.pdf (Last accessed February 4, 2017).

7. Davies E, Higginson I (eds): Palliative Care. Copenhagen: WHO, 2004.

8. Connor SR, Bermedo MCS (eds): Global Atlas of Palliative Care at the End of Life. Geneva, Switzerland, WHO, 2014.

9. Wild D, Grove A, Martin M, et al.: Principles of Good Practice for the Translation and Cultural Adaptation Process for Patient-Reported Outcomes (PRO) Measures: Report of the ISPOR Task Force for Translation and Cultural Adaptation. Value Health 2005;8:94-104.

10. National POLST Paradigm Task Force: http://polst.org/ governing-board (Last accessed January 6, 2018).

11. Appelbaum PS: Assessment of patients' competence to consent to treatment. N Engl J Med 2007;357:1834-1840.

12. Streiner DL: Being inconsistent about consistency: When coefficient alpha does and doesn't matter. J Pers Assess 2003;80:217-222.

13. POLST Legislative Guide 2014: http://polst.org/2014-0220-polst-legislative-guide-final-2 (Last accessed February 3, 2017).

14. Wilkinson A, Wenger N, Shugarman LR: Literature Review on Advance Directives. 2007. http://aspe.hhs.gov/ daltcp/reports/2007/advdirlr.htm (Last accessed June 1, 2013).

15. Andorno R, Biller-Andorno N, Brauer S: Advance health care directives: Towards a coordinated European policy? Eur J Health Law 2009;16:207-227.

16. Brauer S, Biller-Andorno N, Andorno R: Country Reports on Advance Directives. 2008. https://www.ethik.uzh.ch/ dam/jcr:00000000-14d5-886d-ffff-fffff1488f30/Country_ Reports_AD.pdf (Last accessed August 14, 2017).
17. Dantas E: Advance directives and living wills: The role of patient's autonomy in the Brazilian experience. Med Law 2013;32:459-480.

18. Fagerlin A, Schneider CE: Enough. The failure of the living will. Hastings Cent Rep 2004;34:30-42.

19. Castillo LS, Williams BA, Hooper SM, et al.: Lost in translation: The unintended consequences of advance directive law on clinical care. Ann Intern Med 2011;154:121128.

20. Lo B, Steinbrook R: Resuscitating advance directives. Arch Intern Med 2004;164:1501-1506.

21. Pedraza SL, Culp S, Knestrick M, et al.: Association of physician orders for life-sustaining treatment form use with end-of-life care quality metrics in patients with cancer. J Oncol Pract 2017;13:e881-e888.

22. Wenger N, Shugarman LR, Wilkinson A: Advance Directives and Advance Care Planning: Report to Congress. ASPE. 2008. https://aspe.hhs.gov/pdf-report/advance-directivesand-advance-care-planning-report-congress (Last accessed October 17, 2017).

23. Mokkink LB, Terwee CB, Knol DL, et al.: The COSMIN checklist for evaluating the methodological quality of studies on measurement properties: A clarification of its content. BMC Med Res Methodol 2010;10:22.

24. Mokkink LB, Terwee CB, Patrick DL, et al.: The COSMIN checklist for assessing the methodological quality of studies on measurement properties of health status measurement instruments: An international Delphi study. Qual Life Res 2010;19:539-549.

25. Mokkink LB, Terwee CB, Patrick DL, et al.: The COSMIN study reached international consensus on taxonomy, terminology, and definitions of measurement properties for health-related patient-reported outcomes. J Clin Epidemiol 2010;63:737-745.

26. Mokkink LB, Terwee CB, Patrick DL, et al.: COSMIN checklist manual. 2012. www.cosmin.nl/images/upload/ files/COSMIN\%20checklist\%20manual\%20v9.pdf (Last accessed September 1, 2014).

27. Bracher ESB, Pietrobon R, Eluf-Neto J: Cross-cultural adaptation and validation of a Brazilian Portuguese version of the chronic pain grade. Qual Life Res 2010;19:847-852.

28. Barroso EM, Carvalho AL, Paiva CE, et al.: Translation and cross-cultural adaptation into Brazilian Portuguese of the Vanderbilt Head and Neck Symptom Survey version 2.0 (VHNSS 2.0) for the assessment of oral symptoms in head and neck cancer patients submitted to radiotherapy. Braz J Otorhinolaryngol 2015;81:622-629.

29. Rodrigues GR, Oliveira DS, Foss MP, et al.: Cross-cultural adaptation and validation of the episodic autobiographic memory interview for Brazilian Portuguese. Arq Neuropsiquiatr 2015;73:676-680.

Address correspondence to: Edison I.O. Vidal, $M D, M P H, P h D$ Departamento de Clínica Médica Faculdade de Medicina de Botucatu-UNESP Av. Prof. Mario Rubens Guimaraes Montenegro S/N 18618-687

Botucatu Sao Paulo Brazil

E-mail: eiovidal@fmb.unesp.br 